\title{
Erratum:
}

\section{Translated Article: Resection of a Rare Proximal Radius Malignant Chondrosarcoma (Grade III) Bone Tumor and Reconstruction of the Forearm Functionality: A Case Report}

\author{
Sayed Abdulla Jami' ${ }^{1}$, Shi Jiandang ${ }^{* *}$ (D) \\ 1. Department of Spinal Surgery, General Hospital of Ningxia Medical University, Ningxia Medical University, Yinchuan, Ningxia, China.

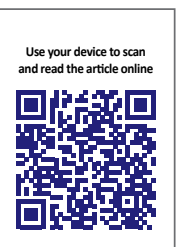 \\ Cftation Jami SA, Jiandang S. Translated Article: Resection of a Rare Proximal Radius Malignant Chondrosarcoma (Grade \\ III) Bone Tumor and Reconstruction of the Forearm Functionality: A Case Report. Journal of Research in Orthopedic Science. \\ 2020; 7(2):79-86. http://dx.doi.org/10.32598/JROSJ.7.2.674.1 \\ Dol of Orghal Artce http://dx.doi.org/10.32598/JROSJ.7.2.674.1 \\ doi http://dx.doi.org/10.32598/JROSJ.7.4.674.1
}

It was found out that the article, entitled "Resection of a Rare Proximal Radius Malignant Chondrosarcoma (Grade III) Bone Tumor and Reconstruction of the Forearm Functionality: A Case Report" [1], was previously published in Chinese in the Orthopedics Journal (ISSN: 1674-8573) in 2018, Volume 9, Number 4, pp. 332-336, doi: 10.3969/j. ISSN.1674-8573.2018.04.017 [2]. To solve the problem, it was referred to the Chinese article in the first page of the current article and revised as the secondary English version of the primary one, based on the 3rd article of "acceptable secondary publication" of ICMJE's guideline overlapping publication [3].

\section{References}

[1] Jami S A, Jiandang S. Resection of a Rare Proximal Radius Malignant Chondrosarcoma (Grade III) Bone Tumor and Reconstruction of the Forearm Functionality: A Case Report. J Res Orthop Sci. 2020; 7(2):79-86. [DOI:10.32598/JROSJ.7.2.674.1]

[2] Jinwen H, Jiandang S, Ningkui N, Zongqiang Y, Longyun W, Huiqiang D. [Reconstruction of forearm support after resection of malignant tumors of proximal radius by butt fusion of distal ulna and radius (with a case report and literature review) (Chinese)] Orthopedics. 2018; 9(4):332-6. [DOI:10.3969/j.issn.1674-8573.2018.04.017]

[3] International Committee of Medical Journal Editors (ICMJE). 2020. Available from: http://www.icmje.org/recommendations/ browse/publishing-and-editorial-issues/overlapping-publications.html

\footnotetext{
* Corresponding Author:

Shi Jiandang, $M D$.

Address: Department of Spinal Surgery, General Hospital of Ningxia Medical University, Ningxia Medical University, Yinchuan, Ningxia, China.

Phone: +86 (137) 09512318

E-mail:shi_jiandang@outlook.com
} 
This Page Intentionally Left Blank 